${ }^{1} M$ Mahomed, ${ }^{2,3}$ S Gimbel, 4,5R Hoek, ${ }^{6} \mathrm{~A}$ Rustagi, ${ }^{7} \mathrm{C}$ Come, ${ }^{8} \mathrm{~L}$ Newman, ${ }^{2} \mathrm{~F}$ Faria, 7.5 Manuel, ${ }^{2,3}$ S Gloyd, ${ }^{9} \mathrm{~N}$ Broutet. 'Instituto Nacional de Saúde (INS), Maputo, Mozambique; ${ }^{2}$ Health Alliance International, Seattle, WA, United States; ${ }^{3}$ University of Washington, Washington, WA, United States; 'University of Washington, Seattle, WA, United States; ${ }^{5}$ Centro de Investigação Operacional da Beira-INS, Beira, Portugal; ${ }^{6}$ Centro de Investigação Operacional da Beira-INS, Covilhã, Portugal; ${ }^{7}$ National Institute of Health, Maputo, Mozambique; ${ }^{8}$ World Health Organization, Geneva, Switzerland; ${ }^{9}$ World Health Organization, Geneva, Switzerland

Background Antenatal syphilis screening and treatment is an effective intervention to reduce perinatal, infant, and maternal morbidity and mortality. Prenatal syphilis screening increased in Manica and Sofala Provinces from < 5\% in 1993 to over $90 \%$ since 2005. This study aimed to (1) estimate the prevalence of congenital syphilis in the cities of Beira and Chimoio, (2) determine differences in congenital syphilis among women treated early in pregnancy, late in pregnancy, and not treated, and (3) identify factors associated with syphilis screening among pregnant women.

Methods Pregnant women presenting at one of six maternities in Beira and Chimoio cities, were recruited and screened with rapid syphilis tests (RST) and, if positive, further screened using Rapid Plasma Reagin (RPR). All live newborns whose mothers tested positive at any point during pregnancy or at birth were RPR screened to detect congenital syphilis. A newborn subsample also had blood and tissue samples taken for IgM and PCR testing to validate the RPR results.

Results Between March 5, 2012 and March 15, 2013, 16,812 women were recruited out of the 19,821 births registered at the participating maternities. Overall, 611 women (3.6\% total) tested positive for syphilis; 498 were syphilis positive by RST at the maternity. An additional 113 women had tested positive at ANC, but were RST negative at delivery. Of the total 405 women who had tested syphilis positive (RST or RPR) and been treated in ANC, their syphilis results at delivery were the following: 286 (71\%) were RST positive, 192 (47\%) were both RST and RPR positive, and 35 (9\%) were RPR positive and RST negative. There was no significant difference in RST/RPR rates between women treated before or after 28 weeks gestation. Of the 16,322 women who were syphilis negative at ANC, 211 (1.26\%) were RPR positive at birth (suggestive of recent infection); of these women, 152 (54\%) were RPR positive. Estimates of the prevalence of and factors associated with congenital syphilis and association with gestational age of treatment are pending, awaiting results of confirmatory testing.

Conclusions The congenital syphilis prevalence rate is substantially lower in this region than in other regions of Mozambique, likely related to years of antenatal syphilis screening. Lifetime positivity of RST was variable, as nearly $30 \%$ of women with positive RST tests in pregnancy were RST negative at birth. In addition, nearly half of women treated adequately remained RPR positive at delivery after treatment.

\section{S14.2 THE CISNE PROJECT: IMPLEMENTATION OF POCT FOR SYPHILIS AND HIV IN ANTENATAL CARE AND REPRODUCTIVE HEALTH SERVICES IN PERU}

\section{doi:10.1136/sextrans-2013-051184.0066}

'P Garcia, ${ }^{2} \mathrm{R}$ Peeling, ${ }^{3} \mathrm{D}$ Mabey, ${ }^{4} \mathrm{~K}$ Holmes, ${ }^{5} \mathrm{C}$ team. ${ }^{1}$ Universidad Peruana Cayetano Heredia, Lima, Peru; 'London School of Hygiene and Tropical Medicine, London, UK; ${ }^{3}$ London School of Hygiene and Tropical Medicine, London, UK; ${ }^{4}$ University of Washington, Seattle, WA, United States, ${ }^{5}$ CISNE team, Lima, Peru

Background Congenital syphilis and syphilis in pregnancy in Peru persist as important public health issues, and improvement of screening/treatment for pregnant women remains challenging. Rapid syphilis testing (RST) allows simple and immediate diagnosis and treatment at a single clinic visit and could increase screening and treatment coverage reducing the incidence of stillbirth and congenital syphilis and generate in the long term a sustainable cost effective intervention.
Methods We tested the feasibility, performance, impact and costeffectiveness of implementing RST in an underserved urban area at a biggest maternity hospital in Peru and a network of 16 peripheral health centres offering prenatal care in a periurban poor area in $\mathrm{Cal}-$ lao-Ventanilla, Peru. RST (integrated with HIV rapid test: the "two for one") were offered at the first prenatal visit (ANC), at delivery and within miscarriage/abortion services.

Results Data from the baseline pre-implementation evaluation revealed limited coverage of screening and treatment services for maternal syphilis and a complex and inefficient system for ANC. RST was started in January 2010. Overall success of implementation was measured by rates of maternal syphilis screening and treatment coverage, partner treatment, and acceptability of RST among providers and patients. We also performed a cost-effectiveness analysis of RST against the Rapid Plasma Reagin (RPR). Attention was paid to the process of dissemination and transfer activities to the Ministry of Health of Peru, through the involvement of both the National Program of STIs and HIV and the Reproductive Health Program. National guidelines have been modified, and recommend the use of both tests, RST and rapid HIV testing in the screening of pregnant women.

Conclusions RST implementation was feasible, successful, acceptable and cost effective. Its introduction catalysed improvements in the quality of care, and by the end of the project it has been introduced in the country as a National policy.

\section{S14.3 EXPLAINING INACTION: THE POLITICS OF CONGENITAL SYPHILIS AND THE GLOBAL HEALTH AGENDA}

doi:10.1136/sextrans-2013-051184.0067

1' Bump, ${ }^{2} \mathrm{~N}$ Salisbury. 'Georgetown University, Washington, DC, United States; ${ }^{2} \mathrm{PATH}$, Seattle, WA, United States

Background If disease burden were directly correlated with priority on the global health agenda, then congenital syphilis would have a high position. But even though congenital syphilis adversely affects around 650,000 pregnancies and newborns annually and is relatively simple to diagnose and treat, it is largely absent from high-level discussions of reproductive and child health and is not a major programmatic focus at prominent global health organisations. We sought to examine explanations for the low prioritisation of congenital syphilis and use our findings to inform framing strategies to improve the placement of congenital syphilis on the global health agenda.

Methods First, we reviewed published literature on congenital syphilis to construct a basic narrative, identify trends, and identify important stakeholders. Second, we conducted semi-structured interviews with stakeholders involved with or important to congenital syphilis control to understand the prioritisation of congenital syphilis and attempts to promote it. Third, we surveyed influential representatives of global health institutions to assess their perspectives on the position of congenital syphilis on the global health agenda. Finally, we analysed the data to track changes in priority given to congenital syphilis over time. We then developed political strategies based on these data.

Results Based on our literature review and key informant interviews, we develop framing strategies, identify potential political allies, and suggest pathways advocates can use to increase the prominence of congenital syphilis on the global health agenda.

Conclusions Although it is an important cause of stillbirth and neonatal death, congenital syphilis is largely invisible on the global health agenda. Past efforts to raise awareness have had limited impact. Future efforts toward elimination of congenital syphilis could benefit greatly from increased attention to framing strategies and alliances with related issues in global health. 